\title{
Synthesis and Antitubercular Acitivity of New Imidazo $[2,1-B][1,3,4]$ Thiadiazole-Phenothiazine Derivatives
}

\author{
Sunil Makwane ${ }^{1}$ and Rajiv Dua ${ }^{2 *}$ \\ ${ }^{1}$ Department of Chemistry, Dr. Harisingh Gour University, India \\ ${ }^{2}$ Department of Chemistry, Government of Madhya Pradesh, India
}

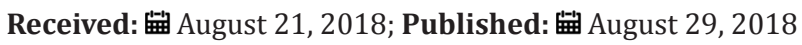

*Corresponding author: Rajiv Dua, Department of Chemistry, State Forensic Science Laboratory, Department of Home (Police), Government of Madhya Pradesh, India

\begin{abstract}
New series of 10-(2-Styryl-5,6-dihydro-imidazo[2,1-b] [1,3,4] thiadiazole-6-yl)-10H-phenothiazine were synthesized by cyclisation of various carboxylic acid with thiosemicarbazide in presence of sulphuric acid was to get compound 1 . Another way phenothiazine treated with chloroacetyl chloride yielded compound 2. Further cyclisation of compound 1 and 2 followed by refluxation about $18 \mathrm{hrs}$ to get the final products 3 and 3a-3i of the series. The structures of compounds were confirmed by IR, ${ }^{1} \mathrm{H}-\mathrm{NMR},{ }^{13} \mathrm{C}$ NMR and mass spectroscopy and by chemical analysis. All the final synthesized compounds 3 and $3 \mathrm{a}-3 \mathrm{i}$ were screened for their antitubercular activity screened against M. tuberculosis H37 Rv.
\end{abstract}

Keywords: Thiadiazole; Phenothiazine; Thiadiazole; Antitubercular Activity

Graphical Abstract

Scheme 1.

\section{Introduction}

Tuberculosis (TB) is one of the dominant killer diseases [1] and it causes huge amount of human deaths despite the availability of more than 20 anti TB drugs and the Bacille Calmette Guerin (BCG) anti TB vaccine [2]. The emergence of the extensively drug-resistant tuberculosis (XDR-TB) and multidrug-resistant tuberculosis (MDR-TB), against which the traditional anti-TB drugs show limited efficacy,[3] further cause serious problem in TB control. With a population of 1.32 billion, India has the highest burden of tuberculosis (TB) and drug resistant TB (DR-TB) in the world. The Global TB Report 2017 published by World Health Organization (WHO) estimates that India contributes 27\% (2.79 million) and 25\% (147 000) of the global burden of TB and multi-drug resistant TB (MDR-TB), respectively. The Revised National Tuberculosis Control Programme (RNTCP) has notified 1.94 million patients in 2016. India has been locating and treating MDR-TB patients since 2007 and achieved complete geographical coverage of programmatic management of drug-resistant TB (PMDT) services in 2013 [4]. In the past years, the literature is enriched with progressive finding about the synthesis and pharmacological actions of fused heterocycles [5]. In the field of synthetic organic chemistry nitrogen- sulphur heteroatom containing aromatic molecules particularly 10-H-phenothiazine and 1,3,4-thiadiazole are becoming more popular as an area of research and provides a most valuable molecular template for the development of new molecule that can interact with a wide variety of biochemical processes. They have been shown to possess a broad spectrum of pharmacological activities such as anti-tubercular [6-7] anti-tumour [8] anti-inflammatory [9] antihyperlipidemic [10], cytotoxic [11], antimicrobial [12] and antiproliferative [13] agents. In continuation in our aim synthesis of new bioactive molecule by incorporating phenothiazine and 1,3,4 heterothiadiazole moieties in a single molecular framework, both molecules have broad biological spectrum such as antibacterial $[14,15]$, antifungal [16,17] anticancer [18,19] anticonvulsant $[20,21]$, antitubercular [22,23] and anti-inflammatory [24,25] herein, we carry out the synthesis and antimicrobial evaluation of some new synthesized molecule. Number of molecules have been claimed by researchers Imidazo [2,1-b]-1,3,4-thiadiazole all around the world because of its excellent biological profile. We have decided to synthesize a new series of 10-(2-Phenyl-imidazo [2,1-b] $[1,3,4]$ thiadiazol-6-yl)-10H-phenothiazine shown in Scheme 1 . The 
starting material, thiosemicarbazide undergoes cyclodehydration of acyl thiosemicarbazides treated with in situ by heating the various carboxylic acid in presence of $\mathrm{H}_{2} \mathrm{SO}_{4}$ yielded compound 1 , 5-Phenyl-[1,3,4]thiadiazol-2-ylamine. In another separate reaction $10-\mathrm{H}$ phenothiazine treated with chloroacetyl chloride yielded compound 2, 2-Chloro-1-phenothiazin-10-yl-ethanone. Further condensation reaction of compound 2 and compound 1 under reflux in dry ethanol 18 hrs yielded compound 3, 10-(2-Phenyl- imidazo[2,1-b] [1,3,4] thiadiazol-6-yl)-10H-phenothiazine, further compounds (3a-3i) synthesized by similar method as reported earlier. The structure of compounds 1 and (1a-1i), compound 2, and compound 3 and (3a-3i) were confirmed by IR, ${ }^{1} \mathrm{H} N M R,{ }^{13} \mathrm{C}$ NMR, mass and chemical analysis. All the compounds 3 and $3 a-3 i$ were screened for their antitubercular activity screened against $\mathrm{M}$. tuberculosis H37 Rv (Scheme 1).

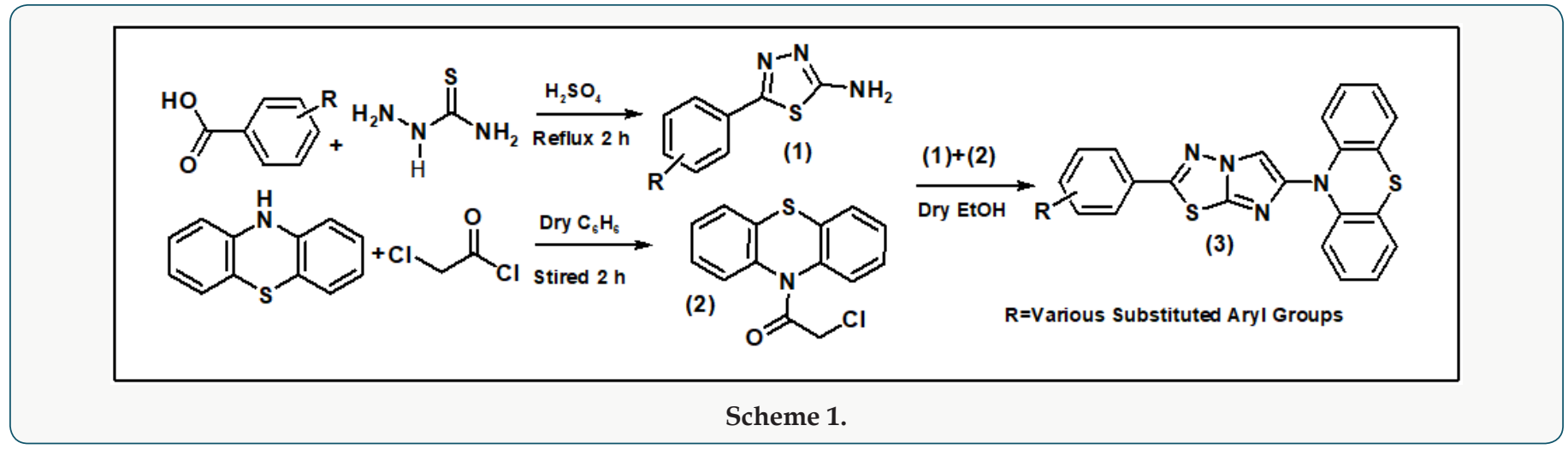

\section{Materials and Methods}

All the chemicals and reagents were of analytical grade of sigma Aldrich, Merck, Chemi-loba and Himedia. The reagents and solvents were purified before using by standard methods. Melting points were taken in open capillaries and are uncorrected. Progress of reaction was monitored at various stages by silica gel-G coated TLC plates using $\mathrm{MeOH}: \mathrm{CHCl}_{3}$ system. The spot was visualized by exposing dry plate at iodine vapour chamber and fluorescent indicator F 254 UV chamber. IR spectra were recorded in $\mathrm{KBr}$ disc on a Schimadzu 8201 PC, FTIR spectrophotometer ( $v$ max in $\mathrm{cm}^{-1}$ ) and ${ }^{1} \mathrm{H}$ NMR and ${ }^{13} \mathrm{C}$ NMR spectra were measured on a Brucker DRX300 spectrometer in $\mathrm{CDCl}_{3}$ at 500 and $75 \mathrm{MHz}$ respectively using TMS as an internal standard. All chemical shifts were reported on $\delta$ scale. The mass spectra were recorded on a Jeol SX-102 GC-MS mass spectrometer. Elemental analyses were performed on a Carlo Erba-1108 analyzer. The analytical data of all the compounds were highly satisfactory. All the synthesized compounds were purified by column chromatography using Merck silica Gel 60 (230-400 Mesh). The reagent grade chemicals were purchased from the commercial sources.

\section{Synthesis of 5-Phenyl- $[1,3,4]$ thiadiazol-2-ylamine}

Equimolar mixture of thiosemicarbazide $(0.004$ mole $)$ and benzoic acid $\left(0.004\right.$ mole) in presence of $\mathrm{H}_{2} \mathrm{SO}_{4}$ in dry ethanol $(25 \mathrm{ml})$ was refluxed on a water bath for about $2 \mathrm{hrs}$ TLC was used to check reaction progress, then mixture was removed and poured in crushed ice to get a white precipitate, compound 1. A solid product was obtained which was purified over a silica gel column using chloroform: methanol (8:2 v/v) mixture as eluant. The elute was concentrated to get a solid product which was recrystallized from ethanol to yielded compound 1: White crystalline solid. M.P. 223$225{ }^{\circ} \mathrm{C}$,Yield 70\%, IR( $\left.v \operatorname{max~cm}^{-1}\right): 1430\left(v_{\mathrm{C}-\mathrm{C}}\right), 1070\left(v_{\mathrm{C}-\mathrm{N}}\right) 763\left(v_{\mathrm{C}-\mathrm{S}}\right)$, $1454\left(v_{\mathrm{C}=\mathrm{C}}\right), 1585\left(v_{\mathrm{N}=\mathrm{C}}\right), 3378\left(v_{-\mathrm{NH} 2}\right),{ }^{1} \mathrm{H}$ NMR: $\delta(\mathrm{ppm}) 4.87(2 \mathrm{H}, \mathrm{s}$, $\mathrm{NH}_{2}$ ) , $7.29-7.73$ (5H, m, Ar-H), ${ }^{13} \mathrm{C}$ NMR : $\delta$ (ppm)126.9-131.01(C of aromatic ring), $169.4,163.8\left(\mathrm{C}_{2}, \mathrm{C}_{5}\right.$ of thiadiazole ring), Anal. Calcd. for $\mathrm{C}_{8} \mathrm{H}_{7} \mathrm{~N}_{3} \mathrm{~S}: \mathrm{C}, 54.22, \mathrm{H}, 3.98, \mathrm{~N}, 23.71 \%$ found $\mathrm{C}, 54.09, \mathrm{H}, 3.70, \mathrm{~N}$, 23.40\%; MS $177.03\left(\mathrm{M}^{+}\right)$.

The compounds 1a-1i were synthesized by the similar method as reported earlier.

a) 5-(2-chloro-phenyl)-[1,3,4]thiadiazole-2-ylamine: M.P.229-230 ${ }^{\circ}$ C, Yield 72\%, IR ( $v$ max cm $\left.{ }^{-1}\right)$ : $1433\left(v_{\mathrm{C}-\mathrm{C}}\right), 1072\left(v_{\mathrm{C}}\right.$ $\left.{ }_{N}\right), 780\left(v_{C-S}\right), 1541\left(v_{C=C}\right), 1587\left(v_{N=C}\right), 745\left(v_{C-C l}\right) 3380\left(v-N_{2}\right),{ }^{1} \mathrm{H}$ NMR $\delta(\mathrm{ppm}) 4.73\left(2 \mathrm{H}, \mathrm{s}, \mathrm{NH}_{2}\right) 7.27-8.18(4 \mathrm{H}, \mathrm{m}, \mathrm{Ar}-\mathrm{H}),{ }^{13} \mathrm{C}$ NMR: $\delta(\mathrm{ppm})$ 127.5-133.1(C of aromatic ring),163.3,169.4 $\left(\mathrm{C}_{2} \mathrm{C}_{5}\right.$ of thiadiazole ring), Anal. Calcd. for $\mathrm{C}_{8} \mathrm{H}_{6} \mathrm{ClN}_{3} \mathrm{~S}: \mathrm{C}, 45.39, \mathrm{H}, 2.86$, $\mathrm{N}, 19.85 \%$ found C,45.09, H, 2.70, N,19.40\%; MS $211.01\left(\mathrm{M}^{+}\right)$

b) 5-(3-chloro-phenyl)-[1,3,4]thiadiazole-2-ylamine: M.P.228-230 ${ }^{\circ} \mathrm{C}$, Yield 69\%, IR( $v$ max cm $\left.\mathrm{cm}^{-1}\right): 1429\left(v_{\mathrm{C}-\mathrm{C}}\right), 1069$ $\left(v_{\mathrm{C}-\mathrm{N}}\right), 779\left(v_{\mathrm{C}-\mathrm{S}}\right), 1537\left(v_{\mathrm{C}=\mathrm{C}}\right), 1587\left(v_{\mathrm{N}=\mathrm{C}}\right), 737\left(v_{\mathrm{C}-\mathrm{Cl}}\right) 3382$ $\left(v-{ }_{\mathrm{NH} 2}\right),{ }^{1} \mathrm{HNMR}: \delta(\mathrm{ppm}) 4.75\left(2 \mathrm{H}, \mathrm{s}, \mathrm{NH}_{2}\right), 7.35-7.68(4 \mathrm{H}$, $\mathrm{m}, \mathrm{Ar}-\mathrm{H}),{ }^{13} \mathrm{C}$ NMR : $\delta$ (ppm) 126.91-31.01 (C of aromatic ring),169.2,162.9 $\left(\mathrm{C}_{2} \mathrm{C}_{5}\right.$ of thiadiazole ring), Anal. Calcd. for $\mathrm{C}_{8} \mathrm{H}_{6} \mathrm{ClN}_{3} \mathrm{~S}: \mathrm{C}, 45.39, \mathrm{H}, 2.86, \mathrm{~N}, 19.85 \%$ found C, 45.19, H, 2.65, N, 19.49\%; MS $211.0\left(\mathrm{M}^{+}\right)$

c) 5-(4-chloro-phenyl)-[1,3,4]thiadiazole-2-ylamine: M.P.230-232 ${ }^{\circ}$ C,Yield73\%, IR( $v$ max cm $\left.{ }^{-1}\right)$ : $1427\left(v_{C-C}\right), 1050\left(v_{C-}\right.$ $\left.{ }_{N}\right), 781\left(v_{\mathrm{C}-\mathrm{S}}\right), 1539\left(v_{\mathrm{C}=\mathrm{C}}\right), 1588\left(v_{\mathrm{N}=\mathrm{C}}\right), 749\left(v_{\mathrm{C}-\mathrm{Cl}}\right), 3383\left(v_{-\mathrm{NH} 2}\right)$, ${ }^{1}$ HMNR: $\delta(p p m) 4.89$ (2H, s, NH ${ }_{2}$ ), 7.73-7.85 (4H, m, Ar-H), ${ }^{13} \mathrm{C}$ 
NMR: $\delta$ (ppm) 129.1-135.6 (C of aromatic ring), 163.4,168.8( $\mathrm{C}_{2} \mathrm{C}_{5}$ of thiadiazole ring), Anal. Calcd. for $\mathrm{C}_{8} \mathrm{H}_{6} \mathrm{ClN}_{3} \mathrm{~S}: \mathrm{C}, 45.39, \mathrm{H}$, 2.86, N, 19.85\% found C, 45.19, H,2.61, N, 19.38\%; MS 211.02 $\left(\mathrm{M}^{+}\right)$

d) 5-(2-bromo-phenyl)-[1,3,4]thiadiazole-2-ylamine: M.P.228-230 ${ }^{\circ}$ C,Yield 68\%, IR ( $v$ max cm$\left.~^{-1}\right): 1426\left(v_{\mathrm{C}-\mathrm{C}}\right), 1055$ $\left(v_{\mathrm{C}-\mathrm{N}}\right), 768\left(v_{\mathrm{C}-\mathrm{S}}\right), 1545\left(v_{\mathrm{C}=\mathrm{C}}\right), 1640\left(v_{\mathrm{N}=\mathrm{C}}\right), 545\left(v_{\mathrm{C}-\mathrm{Br}}\right) 3386\left(v_{\text {. }}\right.$ $\left.{ }_{\mathrm{NH} 2}\right)$, ${ }^{1} \mathrm{HNMR}: \delta(\mathrm{ppm}) 4.80\left(2 \mathrm{H}, \mathrm{s}, \mathrm{NH}_{2}\right)$ 7.25-7.89 (4H, m, Ar$\mathrm{H}),{ }^{13} \mathrm{C}$ NMR : $\delta$ (ppm) 163.1,169.5 ( $\mathrm{C}_{2} \mathrm{C}_{5}$ of thiadiazole ring), 120.7- 132.1(C of aromatic ring), Anal. Calcd. for $\mathrm{C}_{8} \mathrm{H}_{6} \mathrm{BrN}_{3} \mathrm{~S}$ : C, 37.52, H, 2.36, N, 16.41\% found C, 37.18, H, 2.21, N, 16.35\%; MS $254.94\left(\mathrm{M}^{+}\right)$.

e) 5-(3-bromo-phenyl)-[1,3,4]thiadiazole-2-ylamine: M.P. 229-230 ${ }^{\circ}$ C, Yield 67\%, IR ( $v \operatorname{max~cm}^{-1}$ ): $1431\left(v_{\mathrm{C}-\mathrm{C}}\right), 1052$ $\left(v_{\mathrm{C}-\mathrm{N}}\right), 767\left(v_{\mathrm{C}-\mathrm{S}}\right), 1540\left(v_{\mathrm{C}=\mathrm{C}}\right), 1646\left(v_{\mathrm{N}=\mathrm{C}}\right), 536\left(v_{\mathrm{C}-\mathrm{Br}}\right), 3388$ $\left(v_{-\mathrm{NH} 2}\right),{ }^{1} \mathrm{H}$ NMR: $\delta(\mathrm{ppm}) 4.84\left(2 \mathrm{H}, \mathrm{s}, \mathrm{NH}_{2}\right), 7.31-7.64(4 \mathrm{H}$, $\mathrm{m}, \mathrm{Hz}, \mathrm{Ar}-\mathrm{H}),{ }^{13} \mathrm{C}$ NMR: $\delta$ (ppm): 118.1-132.9(C of aromatic ring),164.08,168.9 $\left(\mathrm{C}_{2} \mathrm{C}_{5}\right.$ of thiadiazole ring), Anal. Calcd. for $\mathrm{C}_{8} \mathrm{H}_{6} \mathrm{BrN}_{3} \mathrm{~S}: \mathrm{C}, 37.52, \mathrm{H}, 2.36, \mathrm{~N}, 16.41 \%$ found C, 37.28, $\mathrm{H}, 2.24$, N, 16.25\%; MS $254.82\left(\mathrm{M}^{+}\right)$.

f) 5-(4-bromo-phenyl)-[1,3,4]thiadiazole-2-ylamine: M.P. $231-233{ }^{\circ} \mathrm{C}$, Yield 69\%, IR ( $v$ max cm$\left.{ }^{-1}\right): 1429\left(v_{\mathrm{C}-\mathrm{C}}\right), 1041$ $\left(v_{\mathrm{C}-\mathrm{N}}\right), 766\left(v_{\mathrm{C}-\mathrm{S}}\right), 1543\left(v_{\mathrm{C}=\mathrm{C}}\right), 1642\left(v_{\mathrm{N}=\mathrm{C}}\right), 541\left(v_{\mathrm{C}-\mathrm{Br}}\right), 3390\left(v_{\text {. }}\right.$ $\left.{ }_{\mathrm{NH} 2}\right)$, ${ }^{1} \mathrm{H}$ NMR: $\delta(\mathrm{ppm}) 4.79\left(2 \mathrm{H}, \mathrm{s}, \mathrm{NH}_{2}\right), 7.68-7.79(4 \mathrm{H}, \mathrm{m}, \mathrm{Ar}-\mathrm{H})$, ${ }^{13} \mathrm{C}$ NMR $: \delta$ (ppm) 124.0-131.0 (C of aromatic ring), 164.1$169.5\left(\mathrm{C}_{2} \mathrm{C}_{5}\right.$ of thiadiazole ring), Anal. Calcd. for $\mathrm{C}_{8} \mathrm{H}_{6} \mathrm{BrN}_{3} \mathrm{~S}: \mathrm{C}$, 37.52, H, 2.36, N, 16.41\% found $\mathrm{C}, 37.24, \mathrm{H}, 2.20, \mathrm{~N}, 16.35 \%$; MS $254.86\left(\mathrm{M}^{+}\right)$.

g) 5-(2-nitro-phenyl)-[1,3,4]thiadiazole-2-ylamine: M.P. $257-259^{\circ} \mathrm{C}$, Yield 78\%, IR ( $v$ max cm$\left.{ }^{-1}\right): 1428\left(v_{\mathrm{C}-\mathrm{C}}\right), 1053$ $\left(v_{\mathrm{C}-\mathrm{N}}\right), 778\left(v_{\mathrm{C}-\mathrm{S}}\right), 1515\left(v_{\mathrm{C}=\mathrm{C}}\right), 1651\left(v_{\mathrm{N}=\mathrm{C}}\right), 1341\left(v_{\mathrm{C}-\mathrm{NO} 2}\right), 3391$ $\left(-v_{\mathrm{NH} 2}\right),{ }^{1} \mathrm{H}$ NMR: $\delta(\mathrm{ppm}) 4.90\left(2 \mathrm{H}, \mathrm{s}, \mathrm{NH}_{2}\right), 7.59-8.27(4 \mathrm{H}, \mathrm{m}, \mathrm{Ar}-$ $\mathrm{H}),{ }^{13} \mathrm{C}$ NMR : $\delta$ (ppm) 127.5-148.3(C of aromatic ring), 164.01, 169.6 $\left(\mathrm{C}_{2} \mathrm{C}_{5}\right.$ of thiadiazole ring), Anal. Calcd. for $\mathrm{C}_{8} \mathrm{H}_{6} \mathrm{~N}_{4} \mathrm{O}_{2} \mathrm{~S}: \mathrm{C}$, 43.24, H, 2.72, N, 25.21\% found C,43.14, H, 2.52, N, 25.8\%; MS $222.02\left(\mathrm{M}^{+}\right)$.

h) 5-(3-nitro-phenyl)-[1,3,4] thiadiazole-2-ylamine: M.P. $259-261^{\circ} \mathrm{C}$, Yield 80\%, IR: ( $\left.v \operatorname{max~cm}^{-1}\right): 1426\left(v_{\mathrm{C}-\mathrm{C}}\right), 1048$ $\left(v_{\mathrm{C}-\mathrm{N}}\right), 776\left(v_{\mathrm{C}-\mathrm{S}}\right), 1527\left(v_{\mathrm{C}=\mathrm{C}}\right), 1656\left(v_{\mathrm{N}=\mathrm{C}}\right), 1343\left(v_{\mathrm{C}-\mathrm{N} 02}\right), 3393$ $\left(-v_{\mathrm{NH} 2}\right),{ }^{1} \mathrm{H}$ NMR: $\delta(\mathrm{ppm}) 4.78\left(2 \mathrm{H}, \mathrm{s}, \mathrm{NH}_{2}\right), 7.59-7.91(4 \mathrm{H}, \mathrm{m}, \mathrm{Ar}-$ H), ${ }^{13}$ CNMR: $\delta$ (ppm) 116.3-140.4 (C of aromatic ring), 164.2 $169.3\left(\mathrm{C}_{2} \mathrm{C}_{5}\right.$ of thiadiazole ring), Anal. Calcd. for $\mathrm{C}_{8} \mathrm{H}_{6} \mathrm{~N}_{4} \mathrm{O}_{2} \mathrm{~S}: \mathrm{C}$, 43.24, H, 2.72, N, 25.21\% found C, 43.16, H, 2.62, N, 25.10\%; MS $222.22\left(\mathrm{M}^{+}\right)$.

i) 5-(4-nitro-phenyl)-[1,3,4] thiadiazole-2-ylamine: M.P. $258-260^{\circ} \mathrm{C}$, Yield 79\%, IR $\left(v \operatorname{max~cm}{ }^{-1}\right)$ : $1432\left(v_{\mathrm{C}-\mathrm{C}}\right), 1055\left(v_{\mathrm{C}-}\right.$ $\left.{ }_{\mathrm{N}}\right), 771\left(v_{\mathrm{C}-\mathrm{S}}\right), 1522\left(v_{\mathrm{C}=\mathrm{C}}\right), 1655\left(v_{\mathrm{N}=\mathrm{C}}\right), 1340\left(v_{\mathrm{C}-\mathrm{NO} 2}\right), 3395\left(v_{-\mathrm{NH} 2}\right)$,
${ }^{1} \mathrm{H}$ NMR: $\delta(\mathrm{ppm}) 4.81\left(2 \mathrm{H}, \mathrm{s}, \mathrm{NH}_{2}\right), 7.71-8.27(4 \mathrm{H}, \mathrm{m}, \mathrm{Ar}-\mathrm{H}),{ }^{13} \mathrm{C}$ NMR: $\delta$ (ppm)117.2-140.4( C of aromatic ring), 164.2-168.8 $\left(\mathrm{C}_{2} \mathrm{C}_{5}\right.$ of thiadiazole ring), Anal. Calcd. for $\mathrm{C}_{8} \mathrm{H}_{6} \mathrm{~N}_{4} \mathrm{O}_{2} \mathrm{~S}: \mathrm{C}, 43.24$, $\mathrm{H}, 2.72, \mathrm{~N}, 25.21 \%$ found $\mathrm{C}, 43.26, \mathrm{H}, 2.60, \mathrm{~N}, 25.12 \%$; MS $222.19\left(\mathrm{M}^{+}\right)$.

\section{Synthesis of 2-Chloro-1-phenothiazin-10-yl-ethanone}

Chloroacetyl chloride $(0.06 \mathrm{~mol})$ was added drop wise at 0.5 ${ }^{0} \mathrm{C}$ to phenothiazine $(0.06 \mathrm{~mol})$ in dry benzene $(100 \mathrm{ml})$ and the mixture was stirred for 2 hrs. Reaction progress was checked by TLC during the reaction. After the completion of the reaction, the benzene was distilled off to get a solid product washed with petroleum ether which was purified over a silica gel column using chloroform: methanol $(8: 2 \mathrm{v} / \mathrm{v})$ mixture as eluant. The elute was concentrated to give a product which was recrystallized from ethanol to yielded compound 2. M.P. $190-192^{\circ} \mathrm{C}$, Yield 94\%, IR: ( $v$ $\left.\max \mathrm{cm}^{-1}\right) 1470\left(v_{\mathrm{C}-\mathrm{C}}\right), 2936\left(v_{\mathrm{C}-\mathrm{H}}\right), 1333\left(v_{\mathrm{N}-\mathrm{C}}\right), 1552\left(v_{\mathrm{C}=\mathrm{C}}\right), 2836$ $\left(v_{-\mathrm{CH} 2}\right), 1671\left(v_{\mathrm{C}=0}\right), 685\left(v_{\mathrm{c}-\mathrm{S}-\mathrm{C}}\right), 735\left(v_{\mathrm{C}-\mathrm{Cl}}\right) .{ }^{1} \mathrm{H}$ NMR: $\delta(\mathrm{ppm}) 4.35(2 \mathrm{H}, \mathrm{s}$ acyclic $\mathrm{CH}_{2}$ ), 7.14-7.40 (8H, m, Ar-H), ${ }^{13} \mathrm{C}$ NMR $\delta$ (ppm) 123.1-138.8 ( $\mathrm{C}$ of phenothiazine ring), $165.5\left(\mathrm{C}=\mathrm{O}\right.$ acyclic), $42.2\left(\mathrm{CH}_{2}\right.$ acyclic), Anal. Calcd. for $\mathrm{C}_{14} \mathrm{H}_{10}$ ClNOS: C, 60.98; H, 3.66, N, 5.08, found C, 60.76, H, 3.50, N, 5.01, MS $275.02\left(\mathrm{M}^{+}\right)$.

\section{0-(2-Phenyl-imidazo[2,1-b] [1,3,4] thiadiazol-6-yl)- 10H-phenothiazine}

Equimolar amount of 5-Phenyl- [1,3,4] thiadiazol-2-ylamine, compound 1 (0.004 Mole) and Chloro-1-phenothiazin-10-ylethanone, compound $2(0.004 \mathrm{~mol})$ in ethanol $(20 \mathrm{ml})$ was refluxed on a water bath for about $18 \mathrm{hr}$. After the completion of the reaction, the methanol was distilled off to get a solid product which was purified over a silica gel column using chloroform: methanol (8:2 $\mathrm{v} / \mathrm{v}$ ) mixture as eluant. The elute was concentrated to give a product which was recrystallized from ethanol to yielded compound 3. Light green shinny crystalline solid. Light green crystalline solid, M.P. 210$212^{\circ} \mathrm{C}$, Yield 70\%, IR: $\left(v \operatorname{max~cm}{ }^{-1}\right) 1481\left(v_{\mathrm{C}-\mathrm{C}}\right), 3171\left(v_{\mathrm{C}-\mathrm{H}}\right), 1638\left(v_{\mathrm{C}=\mathrm{N}}\right.$ thiadiazole),1589( $v_{\mathrm{C}=\mathrm{N}} \quad$ imidazole $), 1286\left(v_{\mathrm{N}-\mathrm{C}}\right), 772\left(v_{\mathrm{C}-\mathrm{S}}\right), 1495$ $\left(v_{\mathrm{C}=\mathrm{C}}\right), 681\left(v_{\mathrm{C}-\mathrm{S}-\mathrm{C}}\right.$ phenothiazine). ${ }^{1} \mathrm{H} \quad \mathrm{NMR}: \delta(\mathrm{ppm}) \quad 6.77(1 \mathrm{H}, \mathrm{s}$, imidazole),7.1-7.4 (8H, m, Ar-H phenothiazine),7.45-7.46 (3H, m Ar- $\mathrm{H}$ thiadiazole),8.00(2H, d, J = 8.0, Hz, Ar-H), ${ }^{13} \mathrm{C}$ NMR: $\delta(\mathrm{ppm})$ 125.9-130.4(C of aromatic ring), 124.2, 144.0 and 116.6-128.1(C of phenothiazine), 175.2,164.4 $\left(\mathrm{C}_{2}, \mathrm{C}_{5}\right.$ thiadiazole $), 100.9$ and $150.6(\mathrm{C}$ of imidazole), Anal. Calcd. for $\mathrm{C}_{22} \mathrm{H}_{14} \mathrm{~N}_{4} \mathrm{~S}_{2:} \mathrm{C}, 61.03, \mathrm{H}, 3.03, \mathrm{~N}, 12.94$ $\%$, found C, 61.00, H,3.01, N, 12.74\%; MS $398.06\left(\mathrm{M}^{+}\right)$.

The compounds 3a-3i were synthesized by the similar method as reported earlier.

a. 10-[2-(2-Chloro-phenyl)-imidazo[2,1-b][1,3,4]thiadiazol6-yl]-10H-phenothiazine: M.P. 209-211 ${ }^{0} \mathrm{C}$,Yield 71\%, IR: $\left(v \max \mathrm{cm}^{-1}\right) 1479\left(v_{\mathrm{C}-\mathrm{C}}\right), 3172\left(v_{\mathrm{C}-\mathrm{H}}\right), 1640\left(v_{\mathrm{C}=\mathrm{N}}\right.$ thiadiazole $)$, $1597\left(v_{\mathrm{C}=\mathrm{N}} \quad\right.$ imidazole $), 1280\left(v_{\mathrm{N}-\mathrm{C}}\right), \quad 772\left(v_{\mathrm{C}-\mathrm{S}}\right), \quad 741\left(v_{\mathrm{C}-\mathrm{Cl}}\right)$, $1493\left(v_{\mathrm{C}=\mathrm{C}}\right), 682\left(v_{\mathrm{C}-\mathrm{S}-\mathrm{C}}\right.$ phenothiazine $) .{ }^{1} \mathrm{H}$ NMR: $\delta(\mathrm{ppm}) 6.71(1 \mathrm{H}$, 
s, imidazole), 7.11-7.45 (8H, m, Ar-H phenothiazine), 7.40$7.76\left(4 \mathrm{H}, \mathrm{m}, \mathrm{Ar}-\mathrm{H}\right.$ aromatic ring) ${ }_{13}^{13} \mathrm{C} \mathrm{NMR}: \delta(\mathrm{ppm})$ 127.6133.2 (C of aromatic ring), 124.4,144.2 and 116.7-129.3 (C of phenothiazine),164.7,156.3 ( $\mathrm{C}_{2}, \mathrm{C}_{5}$ thiadiazole $), 100.8$ and 150.4 (C of imidazole), Anal. Calcd. for $\mathrm{C}_{22} \mathrm{H}_{13} \mathrm{Cl} \mathrm{N}_{4} \mathrm{~S}_{2} \mathrm{C}, 61.03$, $\mathrm{H}, 3.03, \mathrm{~N}, 12.94 \%$, found $\mathrm{C}, 61.00, \mathrm{H}, 3.01, \mathrm{~N}, 12.74 \%$; MS $432.03\left(\mathrm{M}^{+}\right)$.

b. 10-[2-(3-Chloro-phenyl)-imidazo[2,1-b][1,3,4] thiadiazol-6-yl]-10H-phenothiazine: M.P. $210-211^{\circ} \mathrm{C}$, Yield 72\%, IR: $\left(v \max \mathrm{cm}^{-1}\right) 1482\left(v_{\mathrm{C}-\mathrm{C}}\right), 3169\left(v_{\mathrm{C}-\mathrm{H}}\right), 1639\left(v_{\mathrm{C}=\mathrm{N}}\right.$ thiadiazole), 1598 ( $v_{\mathrm{C}=\mathrm{N}}$ imidazole), $1284\left(v_{\mathrm{N}-\mathrm{C}}\right), 77\left(v_{\mathrm{C}-\mathrm{S}}\right), 737\left(v_{\mathrm{C}-}\right.$ $\left.{ }_{\mathrm{Cl}}\right), 1491\left(v_{\mathrm{C}=\mathrm{C}}\right), 679 \quad\left(v_{\mathrm{c}-\mathrm{S}-\mathrm{C}}\right.$ phenothiazine $) .{ }^{1} \mathrm{H}$ NMR: $\delta(\mathrm{ppm})$ 6.76 $(1 \mathrm{H}, \mathrm{s}$, imidazole), 7.42-7.71 (3H, m, Ar-H aromatic ring), 7.11-7.44 (8H, m Ar-H penothiazine), $7.95(1 \mathrm{H}, \mathrm{t}, \mathrm{J}=1.5,0.4$ $\mathrm{Hz}, \mathrm{Ar}-\mathrm{H}),{ }^{13} \mathrm{C}$ NMR: $\delta$ (ppm)126.9-131(C of aromatic ring), 124.1,145.1 and 116.6-128.0(C of phenothiazine), 157.7,164.3 $\left(\mathrm{C}_{2}, \mathrm{C}_{5}\right.$ thiadiazole), 100.7 and 150.7 ( $\mathrm{C}$ of imidazole), Anal. Calcd. for $\mathrm{C}_{22} \mathrm{H}_{13} \mathrm{ClN}_{4} \mathrm{~S}_{2:} \mathrm{C}, 61.03, \mathrm{H}, 3.03, \mathrm{~N}, 12.94 \%$, found $\mathrm{C}$, 61.01, H, 3.00, N, 12.84\%; MS $432.02\left(\mathrm{M}^{+}\right)$.

c. 10-[2-(4-Chloro-phenyl)-imidazo[2,1-b][1,3,4]thiadiazol-6yl]-10H-phenothiazine: M.P. $212-214^{\circ} \mathrm{C}$, Yield 70\%, IR:(vmax $\left.\mathrm{cm}^{-1}\right) 1480\left(v_{\mathrm{C}-\mathrm{C}}\right), 3176\left(v_{\mathrm{C}-\mathrm{H}}\right), 1638\left(v_{\mathrm{C}=\mathrm{N}}\right.$ thiadiazole $), 1589$ $\left(v_{\mathrm{C}=\mathrm{N}}\right.$ imidazole $), 1285\left(v_{\mathrm{N}-\mathrm{C}}\right), 770\left(v_{\mathrm{C}-\mathrm{S}}\right), 742\left(v_{\mathrm{C}-\mathrm{Cl}}\right), 1488$ $\left(v_{\mathrm{C}=\mathrm{C}}\right), 682\left(v_{\text {c-s-c }}\right.$ phenothiazine). ${ }^{1} \mathrm{H}$ NMR: $\delta(\mathrm{ppm}) 6.79(1 \mathrm{H}$, $\mathrm{s}$, imidazole),7.68-7.70 (4H, m, Ar-H aromatic ring), 7.127.46 (8H, m Ar-H phenothiazine), ${ }^{13} \mathrm{C}$ NMR : $\delta$ (ppm) 127.2135.7 (C of aromatic ring), 124.4,145.3 and 116.1-128.3(C of phenothiazine),157.7,156.3 $\left(\mathrm{C}_{2}, \mathrm{C}_{5}\right.$ thiadiazole $), 100.6$ and 150.3 (C of imidazole), Anal. Calcd. for $\mathrm{C}_{22} \mathrm{H}_{13} \mathrm{Cl} \mathrm{N}_{4} \mathrm{~S} 2 \mathrm{C}, 61.03, \mathrm{H}$ ,3.03, N, 12.94\%, found C, 61.00, H, 3.02, N, 12.72\%; MS 432.05 $\left(\mathrm{M}^{+}\right)$.

d. $\quad 10$ - $[2-(2$-Bromo-phenyl $)$-imidazo $[2,1-b][1,3,4]$ thiadiazol-6-yl]-10H-phenothiazine: M.P. $211-212^{\circ} \mathrm{C}$ Yield 70\%, IR: (vmax cm $\left.\mathrm{cm}^{-1}\right) 1478\left(v_{\mathrm{C}-\mathrm{C}}\right), 3175 \quad\left(v_{\mathrm{C}-\mathrm{H}}\right), 1637\left(v_{\mathrm{C}=\mathrm{N}}\right.$ thiadiazole), 1588( $v_{\mathrm{C}=\mathrm{N}}$ imidazole), $1279\left(v_{\mathrm{N}-\mathrm{C}}\right), 768\left(v_{\mathrm{C}-\mathrm{S}}\right), 542$ $\left(v_{\mathrm{C}-\mathrm{Br}}\right), 1490\left(v_{\mathrm{C}=\mathrm{C}}\right), 685\left(v_{\mathrm{C}-\mathrm{s}-\mathrm{C}}\right.$ phenothiazine $) .{ }^{1} \mathrm{H}$ NMR: $\delta(\mathrm{ppm})$ 6.73 $(1 \mathrm{H}, \mathrm{s}$, imidazole), 7.37-7.77 $(4 \mathrm{H}, \mathrm{m}, \mathrm{Ar}-\mathrm{H}$ aromatic ring),7.11-7.48 (8H, m Ar-H phenothiazine), ${ }^{13} \mathrm{C}$ NMR: $\delta$ (ppm) 120.7-132.1 (C of aromatic ring),124.5,145.5 and 116.5128.3(C of phenothiazine),156.2,164.5( $\mathrm{C}_{2}, \mathrm{C}_{5}$ thiadiazole), 100.4 and 150.1 (C of imidazole), Anal. Calcd. for $\mathrm{C}_{22} \mathrm{H}_{13} \mathrm{Br}$ $\mathrm{N}_{4} \mathrm{~S}_{2:} \mathrm{C}, 55.35, \mathrm{H}, 2.74, \mathrm{~N}, 11.74 \%$, found $\mathrm{C}, 55.20, \mathrm{H}, 2.52, \mathrm{~N}$, $11.62 \%$, MS $475.96\left(\mathrm{M}^{+}\right)$.

e. 10-[2-(3-Bromo-phenyl)-imidazo[2,1-b][1,3,4]thiadiazol6-yl]-10H-phenothiazine: M.P. $213-214^{\circ} \mathrm{C}$, Yield 69\%, IR:( $v$ max $\left.\mathrm{cm}^{-1}\right) 1480\left(v_{C-\mathrm{C}}\right), 3174\left(v_{\mathrm{C}-\mathrm{H}}\right), 1638\left(v_{\mathrm{C}=\mathrm{N}}\right.$ thiadiazole $), 1590\left(v_{\mathrm{C}=\mathrm{N}}\right.$ imidazole),1281( $\left.v_{\mathrm{N}-\mathrm{C}}\right) 766\left(v_{\mathrm{C}-\mathrm{S}}\right), 540\left(v_{\mathrm{C}-\mathrm{Br}}\right) 1491\left(v_{\mathrm{C}=\mathrm{C}}\right), 683\left(v_{\mathrm{C}-}\right.$ s-c phenothiazine). ${ }^{1} \mathrm{H}$ NMR: $\delta(\mathrm{ppm}) 6.76(1 \mathrm{H}, \mathrm{s}$, imidazole $)$, 7.41-7.61 (3H, m, Ar-H aromatic ring), 7.10-7.49 (8H, m, Ar-H phenothiazine), $7.76(1 \mathrm{H}, \mathrm{td}, \mathrm{J}=1.5, \mathrm{~Hz}, \mathrm{Ar}-\mathrm{H}$ aromatic ring $),{ }^{13} \mathrm{C}$ NMR: $\delta(\mathrm{ppm}) 118.7-133.0$ (C of aromatic ring), 124.6,145.7 and 116.7-128.4 (C of phenothiazine) , 175.1,164.4 $\left(\mathrm{C}_{2}, \mathrm{C}_{5}\right.$ thiadiazole), 100.1 and 150.2(C of imidazole), Anal. Calcd. for $\mathrm{C}_{22} \mathrm{H}_{13} \mathrm{Br} \mathrm{N}_{4} \mathrm{~S}_{2:} \mathrm{C}, 55.35 \%, \mathrm{H}, 2.74, \mathrm{~N}, 11.74$ found $\mathrm{C}, 55.20, \mathrm{H}$, 2.52, N, 11.62\%; MS 475.98( $\left.\mathrm{M}^{+}\right)$.

f. $10-[2-(4-B r o m o-p h e n y l)-i m i d a z o[2,1-b][1,3,4]$ thiadiazol-6-yl]-10H-phenothiazine: M.P. $215-216^{\circ} \mathrm{C}$, Yield $68 \%$, IR: $\left(v \max \mathrm{cm}^{-1}\right) 1482\left(v_{\mathrm{C}-\mathrm{C}}\right), 3171\left(v_{\mathrm{C}-\mathrm{H}}\right), 1636\left(v_{\mathrm{C}=\mathrm{N}}\right.$ thiadiazole), 1594 ( $v_{\mathrm{C}=\mathrm{N}}$ imidazole), $1286\left(v_{\mathrm{N}-\mathrm{C}}\right) 769\left(v_{\mathrm{C}-\mathrm{S}}\right), 538$ $\left(v_{\mathrm{C}-\mathrm{Br}}\right) 1489\left(v_{\mathrm{C}=\mathrm{C}}\right), 688\left(v_{\mathrm{C}-\mathrm{S}-\mathrm{C}}\right.$ phenothiazine $),{ }^{1} \mathrm{H}$ NMR: $\delta(\mathrm{ppm})$ $6.72(1 \mathrm{H}, \mathrm{s}$, imidazole), 7.69-7.78 (4H, m, Ar-H aromatic ring), 7.10-7.49 (8H, m Ar-H penothiazine), ${ }^{13} \mathrm{C}$ NMR: $\delta(\mathrm{ppm})-124-$ 131(C of aromatic ring), 124.2,145.8 and 116.8-128.7 ( $\mathrm{C}$ of phenothiazine), 175.5,164.6 $\left(\mathrm{C}_{2}, \mathrm{C}_{5}\right.$ thiadiazole $), 100.3$ and 150.7 (C of imidazole), Anal. Calcd. for $\mathrm{C}_{22} \mathrm{H}_{13} \mathrm{BrN}_{4} \mathrm{~S}_{2:} \mathrm{C}, 55.35, \mathrm{H}$, $2.74, \mathrm{~N}, 11.74 \%$ found $\mathrm{C}, 55.19, \mathrm{H}, 2.42, \mathrm{~N}, 11.72 \%$; MS 475.99 $\left(\mathrm{M}^{+}\right)$.

g. 10-[2-(2-Nitro-phenyl)-imidazo[2,1-b][1,3,4]thiadiazol6-yl]-10H-phenothiazine: M.P. $215-217^{\circ} \mathrm{C}$, Yield 74\%, IR :( $v$ max $\left.\mathrm{cm}^{-1}\right) 1487\left(v_{\mathrm{C}-\mathrm{C}}\right), 3170\left(v_{\mathrm{C}-\mathrm{H}}\right), 1642\left(v_{\mathrm{C}=\mathrm{N}}\right.$ thiadiazole $), 1598\left(v_{\mathrm{C}=\mathrm{N}}\right.$ imidazole), $1287\left(v_{\mathrm{N}-\mathrm{C}}\right), 770\left(v_{\mathrm{C}-\mathrm{S}}\right), 1343\left(v_{\mathrm{C}-\mathrm{NO} 2}\right) 1494\left(v_{\mathrm{C}=\mathrm{C}}\right), 689$ $\left(v_{\text {C-s-c }}\right.$ phenothiazine $),{ }^{1} \mathrm{H}$ NMR: $\delta$ (ppm) $6.73(1 \mathrm{H}, \mathrm{s}$, imidazole), 7.49-8.35 (4H, m, Ar-H aromatic ring), 7.11-7.48 (8H, m Ar-H phenothiazine), ${ }^{13} \mathrm{C}$ NMR : $\delta$ (ppm) 127.6-148.4 (C of aromatic ring), 124.7,144.9 and 115.9-127.8( C of phenothiazine), 156.5,164.4 $\left(\mathrm{C}_{2}, \mathrm{C}_{5}\right.$ thiadiazole), 100.2 and 150.6 (C of imidazole), Anal. Calcd. for $\mathrm{C}_{22} \mathrm{H}_{13} \mathrm{~N}_{5} \mathrm{O}_{2} \mathrm{~S}_{2:} \mathrm{C}, 59.58, \mathrm{H}, 2.95, \mathrm{~N}$, 15.79\%, found C, 59.38, H, 2.85, N,15.59\%; MS $443.05\left(\mathrm{M}^{+}\right)$.

h. 10-[2-(3-Nitro-phenyl)-imidazo[2,1-b] $[1,3,4]$ thiadiazol6-yl]-10H-phenothiazine (3h): M.P. $216-218^{\circ} \mathrm{C}$, Yield 75 \%,IR: $\left(v \max \mathrm{cm}^{-1}\right)\left(v_{\mathrm{C}-\mathrm{C})} 1483,\left(v_{\mathrm{C}-\mathrm{H}}\right) 3172,1641\left(v_{\mathrm{C}=\mathrm{N}}\right.\right.$ thiadiazole $)$, $1596\left(v_{\mathrm{C}=\mathrm{N}}\right.$ imidazole), $1286\left(v_{\mathrm{N}-\mathrm{C}}\right), 773\left(v_{\mathrm{C}-\mathrm{S}}\right), 1340\left(v_{\mathrm{C}-\mathrm{NO} 2}\right), 1492$ $\left(v_{\mathrm{C}=\mathrm{C}}\right), 685\left(v_{\mathrm{C}-\mathrm{S}-\mathrm{C}}\right.$ phenothiazine $),{ }^{1} \mathrm{H}$ NMR: $\delta(\mathrm{ppm}) 6.68(1 \mathrm{H}$, $\mathrm{s}$, imidazole), 7.58-8.84 (4H, m, Ar-H aromatic ring), 7.107.49 (8H, m Ar-H phenothiazine), ${ }^{13} \mathrm{C}$ NMR : $\delta$ (ppm) 116.4140.5(C of aromatic ring), 124.8, 145.9 and 115.8-128.5(C of phenothiazine), 175.7,164.6 $\left(\mathrm{C}_{2}, \mathrm{C}_{5}\right.$ thiadiazole $), 100.1$ and $150.9 \mathrm{C}$ of imidazole, Anal. Calcd. for $\mathrm{C}_{22} \mathrm{H}_{13} \mathrm{~N}_{5} \mathrm{O}_{2} \mathrm{~S}_{2:} \mathrm{C}, 59.58, \mathrm{H}$, 2.95, N, 15.79 found $\mathrm{C}, 59.40, \mathrm{H}, 2.81, \mathrm{~N}, 15.55$; MS $443.04\left(\mathrm{M}^{+}\right)$.

i. $\quad$ 10-[2-(4-Nitro-phenyl)-imidazo[2,1-b][1,3,4]thiadiazol6-yl]-10H-phenothiazine: M.P. $220-222^{\circ} \mathrm{C}$,Yield 76\%, IR: (vmax $\left.\mathrm{cm}^{-1}\right) 1480\left(v_{\mathrm{C}-\mathrm{C}}\right), 3170\left(v_{\mathrm{C}-\mathrm{H}}\right), 1638\left(v_{\mathrm{C}=\mathrm{N}}\right.$ thiadiazole $), 1592$ $\left(v_{\mathrm{C}=\mathrm{N}}\right.$ imidazole $), 1285\left(v_{\mathrm{N}-\mathrm{C}}\right), 770\left(v_{\mathrm{C}-\mathrm{S}}\right), 1338\left(v_{\mathrm{C}-\mathrm{NO} 2}\right), 1491$ $\left(v_{\mathrm{C}=\mathrm{C}}\right), 685$ ( $v_{\mathrm{C}-\mathrm{s}-\mathrm{C}}$ phenothiazine), ${ }^{1} \mathrm{H}$ NMR: $\delta(\mathrm{ppm}) 6.72(1 \mathrm{H}$, $\mathrm{s}$, imidazole), 7.80-8.33 (4H, m, Ar-H aromatic ring), 7.117.49 (8H, m Ar-H phenothiazine), ${ }^{13} \mathrm{C}$ NMR : $\delta(\mathrm{ppm})$ 117.3140.5( $\mathrm{C}$ of aromatic ring), 124.9,145.6 and 116.8-128 (C of phenothiazine $), 175.1,163.4 \quad\left(\mathrm{C}_{2}, \mathrm{C}_{5} \quad\right.$ thiadiazole $), 100.5$ and 
150.8 (C of imidazole), Anal. Calcd. for $\mathrm{C}_{22} \mathrm{H}_{13} \mathrm{~N}_{5} \mathrm{O}_{2} \mathrm{~S}_{2:} \mathrm{C}, 59.58, \mathrm{H}$, 2.95, N,15.79\%, found C, 59.37, H, 2.81, N, 15.59\%; MS 443.02 $\left(\mathrm{M}^{+}\right)$.

\section{Results and Discussion}

The reaction of thiosemicarbazide and benzoic acid afforded compound 1. The IR spectrum $\left(v \mathrm{~cm}^{-1}\right)$ of compound 1 showed absorption peaks at 1560-1650 for $\left(v_{\mathrm{N}=\mathrm{C}}\right), 760-790$ for $\left(v_{\mathrm{C}-\mathrm{S} \text {, }}\right.$ thiadiazole ring) and peaks at 3350-3430 for $\left(v_{-\mathrm{NH} 2}\right)$. The ${ }^{1} \mathrm{H}$ NMR spectrum $\delta(\mathrm{ppm})$ exhibited the value at 3.9-4.9 $\left(2 \mathrm{H}, \mathrm{s}, \mathrm{NH}_{2}\right)$,7.28$7.95(5 \mathrm{H}, \mathrm{m}, \mathrm{Ar}-\mathrm{H})$ and ${ }^{13} \mathrm{C}$ NMR spectrum gave $\delta(\mathrm{ppm})$ at $163-170$ $\left(\mathrm{C}_{2}, \mathrm{C}_{5}\right.$ of thiadiazole ring) supporting the structure of the compound 1. Similarly, the compounds 1 and $1 \mathrm{a}-1 \mathrm{i}$ have been synthesized by taking the various derivatives of benzoic acid. The IR spectra gave absorptions in the range of $1665-1675 \mathrm{~cm}^{-1}$ while strong signals appeared in the range of $\delta(\mathrm{ppm}) 3.9-4.9$ and 7.39-8.20 in the ${ }^{1} \mathrm{H}$ NMR and $\delta(\mathrm{ppm}) 163-170$ in the ${ }^{13} \mathrm{C}$ NMR spectra supported the formation of compounds 1a-1i respectively. Another reaction was carried out between phenothiazine and chloroacetylchloride to give compound 2. The IR spectrum showed absorptions at $1671 \mathrm{~cm}^{-1}$ due to the presence of carbonyl function, 685 ( $v_{\text {c-s-c }}$ phenothiazine), $735\left(v_{\mathrm{C}-\mathrm{Cl}}\right), 2836\left(v_{-\mathrm{CH} 2}\right)$. In the ${ }^{1} \mathrm{H}$ NMR, strong signals found at 4.35 $\left(2 \mathrm{H}, \mathrm{s}\right.$ acyclic $\left.\mathrm{CH}_{2}\right), 7.14-7.40(8 \mathrm{H}, \mathrm{m}, \mathrm{Ar}-\mathrm{H})$ and ${ }^{13} \mathrm{C}$ NMR spectra gave signals at $\delta(\mathrm{ppm}) 123.1-138.8$ (C of phenothiazine ring), 165.5
( $\mathrm{C}=\mathrm{O}$ acyclic), $42.2\left(\mathrm{CH}_{2}\right.$ acyclic) supporting the confirmation of synthesis of compound 2 . The compounds 1 and $1 \mathrm{a}-1 \mathrm{i}$ on reaction with equimolar amount of compound 2 in methanol gives the compounds 3 and $3 a-3 i$. These compounds showed a characteristic IR absorption in the range of $1580-1600 \mathrm{~cm}^{-1}$ in the IR spectra showing the presence of $\mathrm{N}=\mathrm{C}$ in the imidazole ring. The ${ }^{1} \mathrm{H}$ NMR spectra clearly indicated the presence of one proton in imidazole ring in the range of $\delta(\mathrm{ppm}) 6.68-6.79$. The ${ }^{13} \mathrm{C}$ NMR spectra of compound 3 and 3(a-i) also supported the formation of imidazole ring, $\delta$ (ppm) 100.1- 100.9 and 149.9-150.9 the above structures were supported by fact that the disappearance of $\mathrm{NH}_{2}$ proton and the appearance of $\mathrm{N}=\mathrm{CH}$ proton in the range of $\delta(\mathrm{ppm})$ 6.68-6.79 (cyclic $\mathrm{CH}$ ) in the ${ }^{1} \mathrm{H}$ NMR spectra of compound 3 and 3a-3i. The compounds 2 and $2 \mathrm{a}-2 \mathrm{i}$ and 3 and 3a-3i were synthesized and compounds 3 and 3a-3i were screened for their antitubercular activity screened against M (Scheme 2). tuberculosis H37 Rv. Nitro group containing compounds showed higher activity in the order $(3 \mathrm{~h}>3 \mathrm{i}>3 \mathrm{~g})$ than chloro $(3 \mathrm{~b}>3 \mathrm{a}>3 \mathrm{c})$ or bromo group containing compounds in the order ( $3 e>3 f>3 d$ ). Based on structural activity relationship (SAR), concluded that the activity of compounds depends on electron withdrawing nature of the substituted groups, $\mathrm{NO}_{2}>\mathrm{Cl}>\mathrm{Br}>\mathrm{H}$. The MIC value of the synthesized compounds and standard drug showed in the Table 1

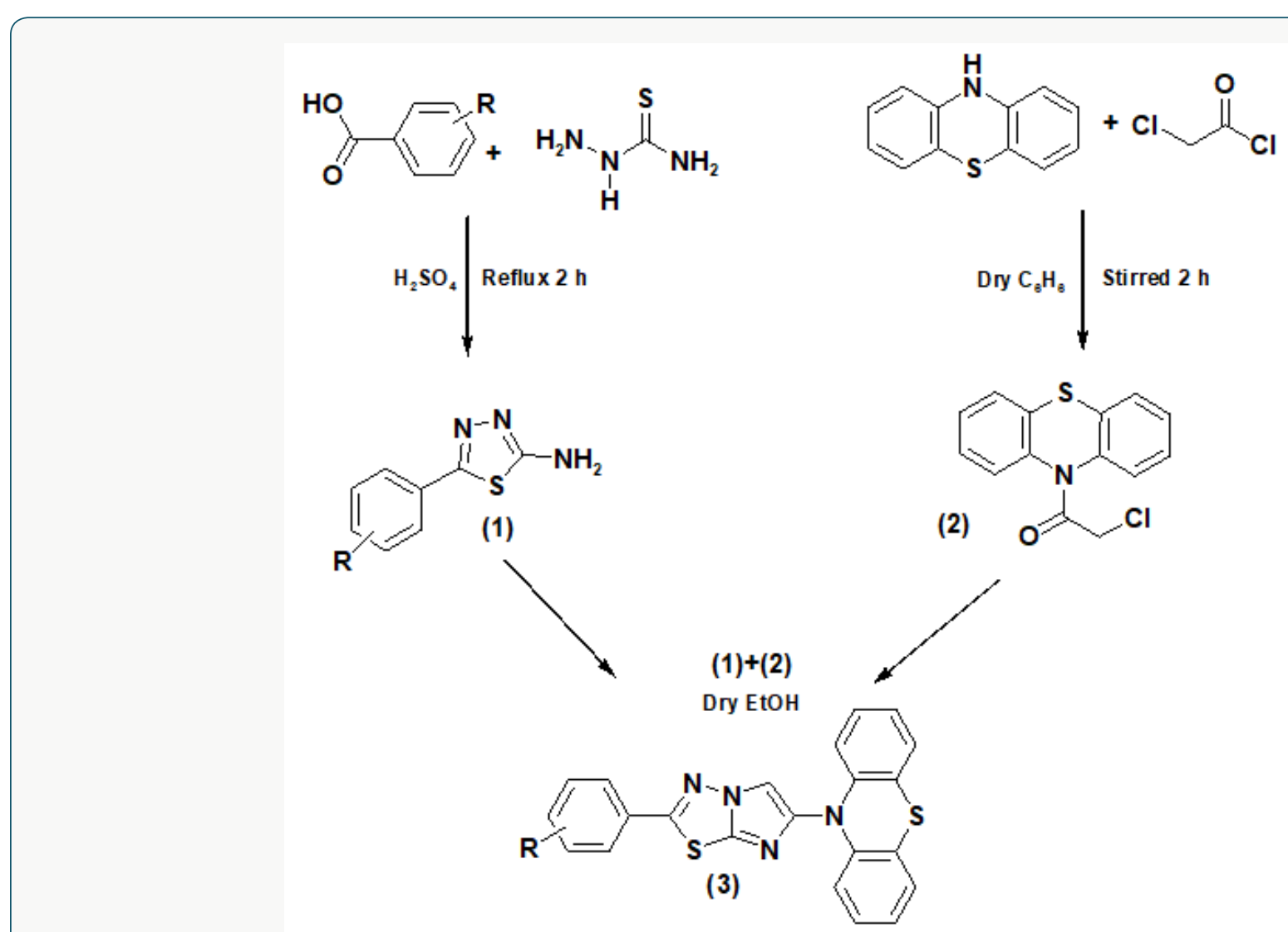

$\mathrm{R}=$ Various Substituted Aryl G roups

Scheme 2: Reaction Scheme. 
Table 1: Characterisation data and Antitubercular activities of compound 3 and 3(a-i).

\begin{tabular}{|c|c|c|c|c|c|c|c|c|}
\hline \multirow[t]{2}{*}{ Comp. } & \multirow[t]{2}{*}{ Ar1 } & \multirow[t]{2}{*}{ Yield \% } & \multirow[t]{2}{*}{ M.P. (OC) } & \multirow[t]{2}{*}{$\begin{array}{l}\text { Molecular } \\
\text { Formulae }\end{array}$} & \multirow[t]{2}{*}{$\begin{array}{c}\text { MS } \\
(\mathrm{M}+)\end{array}$} & \multicolumn{2}{|c|}{$\begin{array}{l}\text { Antitubercular } \\
\text { activity } \\
\text { Inhibition (\%) } \\
\text { (ppm) } \\
\text { M. tuberculosis } \\
\text { H37Rv strain }\end{array}$} & \multirow[t]{2}{*}{$\begin{array}{c}\text { Antitubercular } \\
\text { activity } \\
\text { MIC* } \\
\text { ( } \mu \text { g mL-1) } \\
\text { M. tuberculosis } \\
\text { H37Rv strain }\end{array}$} \\
\hline & & & & & & 25 & 50 & \\
\hline 3 & $\mathrm{C}_{6} \mathrm{H}_{5}$ & 70 & $210-212$ & $\mathrm{C}_{22} \mathrm{H}_{14} \mathrm{~N}_{4} \mathrm{~S}_{2}$ & 398.06 & 22 & 45 & 12 \\
\hline $3 a$ & $2-\mathrm{ClC}_{6} \mathrm{H}_{4}$ & 71 & $209-211$ & $\mathrm{C}_{22} \mathrm{H}_{13} \mathrm{ClN}_{4} \mathrm{~S}_{2}$ & 432.03 & 32 & 79 & 7.5 \\
\hline $3 \mathrm{~b}$ & $3-\mathrm{ClC}_{6} \mathrm{H}_{4}$ & 72 & $210-211$ & $\mathrm{C}_{22} \mathrm{H}_{13} \mathrm{ClN}_{4} \mathrm{~S}_{2}$ & 432.02 & 36 & 80 & 6.5 \\
\hline $3 c$ & $4-\mathrm{ClC}_{6} \mathrm{H}_{4}$ & 70 & $212-214$ & $\mathrm{C}_{22} \mathrm{H}_{13} \mathrm{ClN}_{4} \mathrm{~S}_{2}$ & 432.05 & 32 & 78 & 7 \\
\hline $3 \mathrm{~d}$ & $2-\mathrm{BrC}_{6} \mathrm{H}_{4}$ & 70 & $211-212$ & $\mathrm{C}_{22} \mathrm{H}_{13} \mathrm{BrN}_{4} \mathrm{~S}_{2}$ & 475.96 & 29 & 73 & 10 \\
\hline $3 e$ & $3-\mathrm{BrC}_{6} \mathrm{H}_{4}$ & 69 & $213-214$ & $\mathrm{C}_{22} \mathrm{H}_{13} \mathrm{BrN}_{4} \mathrm{~S}_{2}$ & 475.98 & 30 & 76 & 8.5 \\
\hline $3 \mathrm{f}$ & $4-\mathrm{BrC}_{6} \mathrm{H}_{4}$ & 68 & $215-216$ & $\mathrm{C}_{22} \mathrm{H}_{13} \mathrm{BrN}_{4} \mathrm{~S}_{2}$ & 475.99 & 30 & 75 & 9 \\
\hline $3 \mathrm{~g}$ & $2-\mathrm{NO}_{2} \mathrm{C}_{6} \mathrm{H}_{4}$ & 74 & $215-217$ & $\mathrm{C}_{22} \mathrm{H}^{13} \mathrm{~N}_{5} \mathrm{O}_{2} \mathrm{~S}_{2}$ & 443.05 & 28 & 82 & 5.5 \\
\hline $3 \mathrm{~h}$ & 3- $\mathrm{NO}_{2} \mathrm{C}_{6} \mathrm{H}_{4}$ & 75 & $216-218$ & $\mathrm{C}_{22} \mathrm{H}_{13} \mathrm{~N}_{5} \mathrm{O}_{2} \mathrm{~S}_{2}$ & 443.04 & 27 & 84 & 4 \\
\hline $3 \mathrm{i}$ & $4-\mathrm{NO}_{2} \mathrm{C}_{6} \mathrm{H}_{4}$ & 76 & $220-222$ & $\mathrm{C}_{22} \mathrm{H}_{13} \mathrm{~N}_{5} \mathrm{O}_{2} \mathrm{~S}_{2}$ & 443.02 & 32 & 83 & 5 \\
\hline
\end{tabular}

\section{Antitubercular activity}

The above synthesized compounds were screened against $\mathrm{M}$. tuberculosis (H37Rv strain) using Lowenstein-Jensen (L.J.) Agar method at 50 and $100 \mu \mathrm{g} / \mathrm{mL}$ concentrations. The results were showing in Table 1. The standard antitubercular drugs isoniazid was taken as standards, showed $100 \%$ activity at both the above concentrations. The minimum inhibitory concentration (MIC) values of the synthesized compounds were determined.

\section{Conclusion}

In the conclusion we were successful in the initial hypothesis of synthesizing broad-spectrum antibiotics through experimentation. We report a successful effort to combine pharmacophoric groups; 5-Phenyl- $[1,3,4]$ thiadiazol-2-ylamine and Chloro-1-phenothiazin10-yl-ethanone and the compounds were synthesised in good yield. The structures of compounds were established by FT-IR, ${ }^{1} \mathrm{H}$ NMR, ${ }^{13} \mathrm{CNMR}$ and Mass spectrometry techniques. The synthesized compounds posses antitubercular activity against Mycobacterium tuberculosis H37Rv strain.

\section{Acknowledgement}

The authors are thankful to SAIF, Punjab University, Chandigarh (India) And IISER Bhopal, (M.P.) India for providing spectral and analytical data of the synthesized compounds. We are also grateful to Mr. Vahid-Ul-Hassan, department of Zoology Dr. H.S. Gour University, Sagar [M.P.] for providing help in carrying out the antitubercular screening. We are also thankful to Head, Department of Chemistry, Dr. H. S. Gour Central University Sagar (India) for giving the facilities to carry out the work and UGC-New Delhi (India), for financial assistance as R.G.N.F. fellowship

\section{References}

1. Herzog H (1998) History of tuberculosis. Respiration 65(1): 5-15.

2. Tripathi RP, Tiwari N, Dwivedi N, Tiwari VK (2005) Fighting tuberculosis: an old disease with new challenges. Med Res Rev 25(1): 93-131.

3. Koul A, Arnoult E, Lounis N, Guillemont J, Andries K (2011) The challenge of new drug discovery for tuberculosis. Nature 469 (7331): 483-490.

4. (2017) Global Tuberculosis Report. World Health Organization, Geneva.

5. Tyagi MK, Srivastava SK, Srivastava SD (2014) Synthesis, Characterization of Some Novel Substituted Arylated Derivatives. J Applicable Chem 3(5): 1967-1972.

6. Patel HM, Noolvi MN, Sethi NS, Gadad AK, Cameotra SS (2017) Synthesis and antitubercular evaluation of imidazo[2,1-b] [1,3,4] thiadiazole derivatives. Arabian Journal of Chemistry 10(Supp 1): 996-1002.

7. Ramprasad J, Nayak N, Dalimba U, Yogeshwari P, Sriram D, et al. (2015) Synthesis and biological evaluation of new imidazo[2,1-b] [1,3,4] thiadiazole-benzimidazole derivatives. European Journal of Medicinal Chemistry 95: 49-63.

8. Kumar S, Metikurki B, Bhadauria VS, Clercq ED, Schols D, et al. (2016) Synthesis of imidazo[2,1-b] [1,3,4] thiadiazole derivatives as possible biologically active agents. Acta Poloniae Pharmaceutica n Drug Research 73(4): 913-929.

9. Jadhav VB, Kulkarni MV, Rasal VP, Biradar SS, Vinay MD (2008) Synthesis and anti-inflammatory evaluation of methylene bridged benzofuranyl imidazo [2,1-b] [1,3,4] thiadiazoles. European Journal of Medicinal Chemistry 43(8): 1721-1729.

10. Patel HM, Noolvi MN, Goyal A, Thippeswamy BS (2013) 2,5,6-Trisubstituted Imidazo [2,1 b] [1,3,4] thiadiazoles: Search forantihyperlipidemic agent. European Journal of Medicinal Chemistry 65: 119-133.

11. Kumar S, Hegde M, Gopalakrishnan V, Renuka VK, Ramareddy SA, et al. (2014) 2-(4-Chlorobenzyl)-6-arylimidazo[2,1-b] [1,3,4] thiadiazoles: Synthesis, cytotoxic activity and mechanism of action. European Journal of Medicinal Chemistry 84: 687-697.

12. Alagawadi KR, Alegaon SG (2011) Synthesis, Characterization and Antimicrobial activity evaluation of new 2,4-Thiazolidinediones bearing 
imidazo [2,1-b] $[1,3,4]$ thiadiazole moiety. Arabian Journal of Chemistry 4: 465-472.

13. Romagnoli R, Baraldi PG, Prencipea F, Balzarinib J, Liekensb S, et al. (2015) Design, synthesis and antiproliferative activity of novel heterobivalent hybrids based on imidazo[2,1-b] $[1,3,4]$ thiadiazole and imidazo[2,1b] $[1,3]$ thiazole scaffolds. European Journal of Medicinal Chemistry 101(28): 205-217.

14. Sharma R, Samadhiya P, Srivastava SD, Srivastava SK (2012) Synthesis and biological activity of 4-thiazolidinone derivatives of phenothiazine. ] Serb Chem Soc 77(1): 17-26.

15. Jalhan S, Jindal A, Gupta A, Hemraj (2012) Synthesis, Biological Activities and Chemistry of Thiadiazole Derivatives and Schiff Bases. Asian Journal of Pharmaceutical and Clinical Research 5(3):199-208.

16. Kumar V, Upadhyay N, Manhas A (2015) Designing, syntheses, characterization, computational study and biological activities of silverphenothiazine metal complex. Journal of Molecular Structure 1099: 135-141.

17. Li Q1, Ren J, Dong F, Feng Y, Gu G, et al. (2013) Synthesis and antifungal activity of thiadiazole-functionalized chitosan derivatives. Carbohydrate Research 373: 103-110.

18. Abuhaie CM, Bicu E, Rigo B, Gautret P, Belei D, et al. (2013) Synthesis and anticancer activity of analogues of phenstatin, with a phenothiazine A-ring, as a new class of microtubule-targeting agents. Bioorganic \& Medicinal Chemistry Letters 23(1): 147-152.
19. Azaam MM, Kenawy ER, Badr El din AS, Khamis AA, El Magd MA (2018) Antioxidant and anticancer activities of aminophosphonates containing thiadiazole moiety. Journal of Saudi Chemical Society 22(1): 34-41.

20. Laws ML, Roberts RR, Nicholson JM, Butcher R, Stables JP, et al. (1998) Synthesis, Characterization, and Anticonvulsant Activity of Enaminones. Part 5: Investigations on 3-Carboalkoxy-2- methyl-2,3-dihydroNY-phenothiazin-4[10H)-one Derivatives. Bioorganic \& Medicinal Chemistry 6(12): 2289-2299.

21. Harish KP, Mohana KN, Mallesha L (2013) Synthesis of indazole substituted-1,3,4- thiadiazoles and their anticonvulsant activity. Drug invention today 5(2): $92-99$.

22. Sharma R, Samadhiya P, Srivastava SD, Srivastava SK (2011) Synthesis and biological activity of 2-oxo-azetidine derivatives of Phenothiazine. Org Commun 4(2): 42-51.

23. Talath S, Gadad AK (2006) Synthesis, antibacterial and antitubercular activities of some 7-[4-(5-amino-[1,3,4] thiadiazole-2-sulfonyl)-piperazin-1yl] fluoroquinolonic derivatives. European Journal of Medicinal Chemistry 41(8): 918-924.

24. Sadanandam YS, Shetty MM, Bhaskar Rao A,RambabuY (2009) $10 \mathrm{H}-$ Phenothiazines: A new class of enzyme inhibitors for inflammatory diseases. European Journal of Medicinal Chemistry 44(1):197-202.

25. Palaska E, Sahin G, Kelicen P, Durlu NT, Altinok G (2002) Synthesis and anti-inflammatory activity of 1-acylthiosemicarbazides,1,3,4oxadiazoles,1,3,4-thiadiazoles and 1,2,4-triazole-3-thiones. II Farmaco 57(2): 101-107.

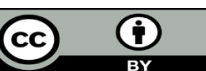

This work is licensed under Creative Commons Attribution 4.0 License

To Submit Your Article Click Here: Submit Article

DOI: $10.32474 /$ AOICS.2018.03.000169

\section{AOICS}

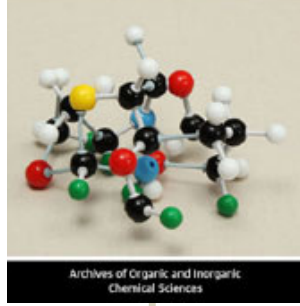

\section{Archives of Organic and Inorganic} Chemical Sciences

\section{Assets of Publishing with us}

- Global archiving of articles

- Immediate, unrestricted online access

- Rigorous Peer Review Process

- Authors Retain Copyrights

- Unique DOI for all articles 